\title{
Crisis financiera del 2008: efecto en las empresas listadas en la Bolsa Mexicana de Valores
}

\section{Aurora Jaramillo Olivares*}

Universidad Autónoma del Estado de México, Centro Universitario, UAEM

\author{
Marcela Jaramillo Jaramillo \\ Universidad Autónoma del Estado de México, Centro Universitario, UAEM
}

(Recibido 23 de junio 2015, aceptado 4 de octubre de 2015.)

\begin{abstract}
Resumen
El objetivo del trabajo es conocer si las empresas que cotizan en la Bolsa Mexicana de Valores sufrieron reacción negativa en el precio de sus acciones derivado de la crisis financiera ocurrida en 2008. La metodología utilizada fue la de estudio de eventos mediante el modelo de mercado y el cálculo de los retornos anormales de la fecha evento. El análisis se llevó a cabo con 87 empresas, de las cuales se obtuvo información de las cotizaciones diarias en un período comprendido de enero 2008 a febrero 2009. Los resultados revelan que las empresas de la BMV no sufren efectos económicos negativos en el precio de sus acciones, sin embargo, el análisis por sector indica, que el grupo de empresas del sector industrial tuvieron resultados negativos. Las limitaciones son el número de empresas de las cuales se pudo obtener cotizaciones diarias y la elección de la fecha y ventanas evento. Este tipo de estudios, apoyarían a gobiernos, instituciones o empresas a actuar ante fenómenos como el analizado, y así, buscar la forma de regularlo para evitar que vuelvan a repetirse.
\end{abstract}

Clasificación JEL: D53, E44, G14.

Palabras clave: Bolsa Mexicana de Valores, Crisis financiera, Reacción de mercado.

\section{The 2008 Financial Crisis: Effect on Listed Companies on the Mexican Stock Exchange}

\section{Abstract}

The objective of this work is to know if the companies listed on the Mexican Stock Exchange, suffered negative reaction in the price of its shares derived from the financial crisis in 2008 . The methodology used was the study of events through the market model and the calculation of the abnormal returns of the event date. The analysis was carried out with 87 companies, of which information was obtained from the daily prices in a period from January 2008 to February 2009. The results reveal that the companies of the BMV do not suffer negative economic effects on the price of their actions; however, the analysis by sector shows that the group of companies in the industrial sector had negative results. The limitations are the number of companies of which we were able to obtain daily contributions and the choice of the date and windows event. This type of studies, support to governments, institutions or companies to act before phenomena as the analyzed, and so find the way to regulate it to avoid a recurrence.

JEL Classification: D53, E44, G14.

Key Words: Mexican Stock Exchange, Financial Crisis, Market Reaction.

* Carretera Toluca-Tejupilco Km. 67.5, Barrio de Santiago, C. P. 51300 Temascaltepec de González, México, México. Tel. 01(722)4272368. Correo Electrónico:aurora_j15 @hotmail.com 


\section{Introducción}

A lo largo de la historia, la economía ha sido una incógnita de carácter cambiante y dinámico que han intentado explicarse investigadores, economistas, inversionistas, accionistas, o simplemente personas sumergidas en este tema por interés propio. Las crisis financieras forman parte de la inestabilidad económica y representan para la economía de los países una serie de constantes cambios; no obstante, muchas veces estos cambios son ocasionados por la población misma, es decir provocan una crisis especulativa, esta ocurre cuando los precios suben por que la gente piensa que van a subir en el futuro (Samuelson y Nordhaus, 2002). De lo anterior se puede afirmar que de una u otra manera el mundo siempre está presenciando una crisis de cualquier tipo, ahora bien retornando a la Gran Recesión que se sufrió en 2008, y de la cual aún se están resintiendo los efectos, sería conveniente analizar el efecto de la crisis financiera mundial del 2008 en las cotizaciones bursátiles de las empresas inscritas en la BMV.

Así, fácilmente se puede deducir que si las crisis han afectado la economía mexicana, seguramente el mercado de valores mexicano, no ha sido ajeno a tan devastadores fenómenos, tal y como lo afirma Tomé (2012).

En el presente trabajo se medirán y analizarán las posibles consecuencias económicas que han tenido los inversores del mercado de capitales en México por la quiebra de Lehman Brothers, ya que fue uno de los bancos de inversión más importantes. Por ello se medirá la reacción de los precios diarios de las acciones, utilizando la metodología de estudio llamada "event studies", esto con el fin de probar si la quiebra de dicho banco tuvo efectos en las cotizaciones. Se ofrece un análisis minucioso de los efectos de la crisis financiera de 2008 en las cotizaciones bursátiles de las empresas que listadas en la BMV, dejando en claro y de manera sustentada el efecto que tuvo este fenómeno sobre los precios de las cotizaciones para este periodo, asimismo se muestra detalladamente, la evolución general de la BMV para las fechas en las que tuvo lugar la crisis, también se visualiza cómo hubiera continuado el mercado en caso de que la crisis no hubiese ocurrido.

\section{2. ¿Qué pasó en 2008?}

Entre diciembre de 2006 y diciembre de 2007, el precio de las casas cayó en un $25 \%$ y se dio inicio a la "crisis inmobiliaria" preparando el escenario para la crisis financiera de 2008 (Barcelata, 2010).

Puede resaltarse que entre tantas quiebras, desfalcos, fraudes, disminución abrupta del empleo, el año 2008 fue exactamente el que marcó profundamente la economía global. Asimismo, cabe mencionar que esta crisis inicialmente fue llamada crisis de las hipotecas "subprime", dichas hipotecas se originaron con excesos en 2007, ocasionando una desestabilización en el sistema financiero, de aquí que se trate de una crisis financiera de alcance mundial (Zúñiga y Castellanos, 2008).

Sin embargo, Llamas (2009) explica "todo reside en que las famosas hipotecas basura no eran la causa, sino el primer síntoma evidente del estallido de la burbuja crediticia que durante más de una década, fue alentada desde los organismos financieros de planificación central". En efecto esto es así, debido a que antes de que se diera la crisis financiera, se dio una crisis hipotecaria, pero como afirma Llamas, esto no era más que una primera causa de lo que estaba por suceder. 
Se pretende mostrar que la crisis no es un fenómeno de carácter accidental, sino que es resultado del conjunto de políticas públicas que han aplicado los gobiernos de Estados Unidos en los últimos años, estableciendo un marco institucional que pone en riesgo el funcionamiento ordenado de su economía y de la economía mundial en el largo plazo.

Asimismo, Llamas (2009) explica lo que sucedió en 2008 de manera concreta y resumida en palabras simples. La burbuja estalló. La inflación comenzó a aumentar, obligando a una subida de tipos de interés que finalmente descubrió lo bueno de las hipotecas "subprime". El precio de la vivienda se desmoronó, los impagos hipotecarios se dispararon, los embargos se sucedieron. Como resultado, se produce la quiebra de los fondos del banco de inversión Bear Stearns en el verano de 2007. El grifo de la financiación interbancaria se cerró. La imposibilidad de hacer frente a la deuda comprometida a corto plazo inició la sucesión de bancarrotas que se extiende hasta hoy en día.

De lo anterior fácilmente se puede concluir que lo que sucedió fue un montón de malas decisiones tomadas por parte de las hipotecarias y bancos, fue exceso de confianza en este mercado, y claramente la economía se aprovechó de esto, y como se puede observar la crisis ha afectado a aquellos que tomaron préstamos hipotecarios y ha provocado pérdidas millonarias y quiebras. Los consumidores en el mundo han dejado de gastar en bienes de consumo y la producción mundial cae como consecuencia, amenazando la estabilidad económica internacional, esto de acuerdo a un informe emitido por el FMI (2015).

\subsection{Efectos de la crisis en México}

Cabe mencionar que en México uno de los más grandes efectos de la crisis financiera ocurrida en 2008 es la disminución de las exportaciones, considerando que el $85 \%$ de lo que México exporta tiene como destino Estados Unidos, de lo cual el $75 \%$ son manufacturas, se espera que los sectores más afectados sean la industria automotriz y la relacionada con insumos para la construcción (Saavedra, 2009). En este sentido, Mattar (2008) ha manifestado que es necesario que México diversifique el destino de sus exportaciones ya que actualmente las que se realizan hacia la Unión Americana representan la tercera parte del PIB mexicano. La dependencia del sector externo mexicano en cuanto al destino de sus exportaciones se puede apreciar en el Cuadro 1.

Cuadro 1. Principales socios comerciales de México (2007).

\begin{tabular}{|c|c|}
\hline País/Región & $\begin{array}{c}\text { COMERCIO TOTAL (Miles de } \overline{\boldsymbol{M}} \text { de } \\
\text { USD) }\end{array}$ \\
\hline Estados Unidos & 21.086 .633 .741 \\
\hline Unión Europea & 1.380 .971 .815 \\
\hline Canadá & 623.377 .088 \\
\hline China & 162.636 .924 \\
\hline Japón & 161.627 .476 \\
\hline África & 83.193 .926 \\
\hline
\end{tabular}

Fuente: López, 2008.

Como se observa en el cuadro anterior, analizando las cifras se deja clara la dependencia económica que México tuvo en ese año con Estados Unidos, de este 
modo se apoya la opinión de Mattar (2008), afirmando que esta dependencia sigue en pie, según un informe publicado por la SAGARPA en 2015.

Cabe destacar que son numerosos los puntos que interrelacionan a estos dos países, pero para efectos de esta investigación, solo se enfatizó en las exportaciones debido al gran porcentaje que representan en el PIB mexicano.

Ahora bien, una vez que ya se conocen los efectos que tuvo la crisis en la economía mexicana, es pertinente hacer hincapié sobre el efecto del fenómeno específicamente en la Bolsa Mexicana de Valores (BMV), esto para cumplir con el objetivo principal de la presente investigación; analizar los efectos de la crisis sobre las cotizaciones de las empresas inscritas en la BMV.

\subsection{Metodología para el análisis del evento}

Basados en la teoría de eficiencia del mercado y con la finalidad de conocer las variaciones positivas o negativas en los precios de las cotizaciones de las empresas, se optó por aplicar la metodología de estudio de eventos conocida como "event studes", tal como se ha mencionado en párrafos precedentes. En este tipo de estudios se mide el impacto de un acontecimiento sobre el valor bursátil de la empresa (Soltani, 2000).

Cabe mencionar que esta metodología es ampliamente aceptada y contrastada por los investigadores, debido a que ha sido utilizada en los últimos años en el campo de las finanzas y la contabilidad (Jaramillo y García 2010).

En esta investigación se parte de la hipótesis de que el mercado es eficiente en su nivel intermedio, ello implica que los precios absorben toda la nueva información dada del exterior. Basarse en esta teoría permite conocer como los agentes que operan en la bolsa hacen uso de esta información a través de cambios en los precios de las acciones.

Siguiendo con la metodología, la medida del impacto económico de un evento puede ser construida estudiando el comportamiento de los retornos anormales de los títulos sobre un periodo de tiempo relativamente corto, a dicho periodo se le denomina ventana evento (Jaramillo y García, 2010).

Entonces lógicamente el paso inicial lo constituye la definición del evento que se quiere analizar, así como la especificación de la fecha en la que se produjo y las ventanas en las cuales se van analizar las rentabilidades anormales de los títulos. Cabe mencionar que para el cálculo de los retornos y rendimientos del mercado y empresas, se utilizó el trabajo realizado por Jaramillo y García (2010).

\section{a) Cálculo de los retornos actuales/reales}

Antes de estimar los retornos anormales tenemos que calcular los retornos actuales o reales de la empresa $i$ en el tiempo $t$ tomando para esto las cotizaciones diarias de cada una de las empresas que cotizan en la Bolsa Mexicana de Valores, de esta manera para calcular la rentabilidad real del mercado se utilizará el IPC (Índice de Precios y Cotizaciones) de la BMV, ya que se le considera como el principal indicador en el análisis del mercado bursátil mexicano. Por lo tanto a continuación se presentan las fórmulas para su cálculo:

$$
R_{i t}=\frac{\left(P_{i t}+P_{i t}-1\right)+D_{i t}}{P_{i t}-1}
$$


Donde:

$R_{i t}=$ rendimiento o retorno observado del título $i$ durante el periodo de tiempo $t$ (diarios)

$P_{i t}=$ precio de la acción de la empresa $i$ en el periodo $t$

$D_{i t}=$ pago de dividendos o dividendo ganado por una acción de la empresa $i$ en el periodo $t$

$Y$, para el cálculo del rendimiento del mercado se utilizó la siguiente fórmula:

$$
R_{m t}=\frac{\left(P_{I P C t}+P_{I P C t}-1\right)}{P_{I P C t-1}}
$$

Donde:

$R_{m t}=$ es el retorno actual del mercado en el tiempo $t$

$P_{I P C t}=$ es el precio diario del índice $I P C$ en el momento $t$

$P_{(I P C t-1)}=$ es el precio diario del índice $I P C$ en el momento $t-1$

\section{b) Cálculo de los retornos anormales}

Los retornos anormales recogerán los movimientos en los precios de los títulos que son específicos de la empresa, tras eliminar aquellos cambios que son relativos al mercado. Tal y como lo dice Jaramillo y García (2010) en la literatura contable existen varios modelos que sirven para calcular los residuos o retornos anormales, pero la mayoría de ellos parten de la base de que el cálculo de los residuos de la expresión anterior se puede expresar en los siguientes términos, para una empresa $i$ y una fecha de evento $t$ el retorno anormal será:

$$
\mu_{i t}=R_{i t}\left(\alpha_{i}+\beta_{i} R_{m t}\right)
$$

Donde:

$\mu_{i} t=$ es el retorno o rentabilidad anormal del título $i$ en el tiempo $t$

$R_{i} t=$ es el retorno o rentabilidad real/actual del título $i$ durante el momento $t$

$R_{m t}=$ es la rentabilidad real del mercado en el momento $t$

$\alpha_{i}, \beta_{i}=$ son los parámetros del modelo del mercado

La estimación de los parámetros se realiza por mínimos cuadrados ordinarios.

\section{c) Cálculo de los retornos esperados}

Cabe señalar que $\hat{\alpha}_{i}, \hat{\beta}_{i}$ son los parámetros estimados del modelo de mercado, lo que claramente lleva a deducir que es necesaria la utilización de dicho modelo tal y como lo hizo Jaramillo y García (2010), así entonces:

$$
\begin{gathered}
E R_{i t}=\alpha_{i}+\beta_{i} R_{m t}+\mu_{i t} \\
E\left(\mu_{i t}\right)=0 \\
\operatorname{Var}\left(\mu_{i} t\right)=\sigma^{2} \mu_{i} t
\end{gathered}
$$

Donde: 
$E R_{i t}=$ es la rentabilidad esperada del título $i$ en el momento $t$, $R_{m} t=$ es la rentabilidad real del mercado en el momento $t$,

$\mu_{i t}=$ es el término residual o el error de predicción del título $i$ en el periodo $t$ con esperanza cero y varianza $\sigma \mu_{i t} \mathrm{y}$

$\alpha_{i}, \beta_{i}=$ son los parámetros a estimar.

Y para calcular la rentabilidad real del mercado como ya se dijo anteriormente se utilizará el IPC (Índice de Precios y Cotizaciones) de la BMV, ya que es el principal indicador del mercado mexicano.

\subsection{Fechas y ventanas evento}

Una cuestión importante a la hora de aplicar con éxito un estudio de evento es la habilidad para identificar con exactitud la fecha en que sucede el acontecimiento cuyo impacto se pretende analizar. Una vez que se realizó una revisión de los trabajos y noticias relacionadas con la "Gran Recesión" se ha efectuado el análisis de los retornos anormales para una fecha evento.

El evento lo constituye la noticia pública del cuarto banco de inversión de Estados Unidos, Lehman Brothers que se declara en banca rota abrumado por las pérdidas en el sector inmobiliario. Esa fecha el 15 de septiembre de 2008, también adoptada por varios trabajos relacionados, como el de Alcaide (2009) y Black (2010). Y efectivamente en esta investigación se optó por elegir esta fecha, debido a que durante el fin de semana que va del 12 al 14 de septiembre ya había rumores de lo que pasaría, y se piensa que el mismo día que se declaró en quiebra, los efectos comenzaron.

Así, como se ha señalado anteriormente, una de las limitaciones de la metodología es el hecho de que los inversores pudieran anticipar el colapso en la economía.

Por lo tanto la fecha de evento es:

1. El 15 de septiembre de 2008, cuando Lehman Brothers el cuarto banco de inversión de Estados Unidos, se declara en bancarrota abrumado por las pérdidas en el sector inmobiliario.

Ahora se especifican los periodos sobre los cuales se analizaran los retornos anormales de las empresas, es decir, las ventanas evento

En este estudio se han utilizado diferentes ventanas de evento que comprenden los siguientes intervalos:

La fecha del 15 de septiembre de $2008(\mathrm{t}=0)$, las ventanas de evento comprenden los días incluidos en los siguientes intervalos $(-10,+10) ;(-9,+9)$; $(-8,+8) ;(-7,+7) ;(-6,+6) ;(-5,+5) ;(-4,+4) ;(-3,+3) ;(-2,+2) ;(-1,+1)$, por lo tanto el número de días de cada periodo serán $21,19,17,15,13,11,9,7,5$ y 3 días respectivamente. El día cero será definido como el día que ocurrió el evento.

\section{Hipótesis del trabajo}

Las personas de Wall Street sintieron más de cerca el pánico que sucumbió a Estados Unidos, sin embargo se dejó resentir todo un cúmulo de acontecimientos en México, acontecimientos que inversores principalmente resintieron al ver como sus acciones comenzaban a perder valor, lo que hace pensar que iniciaron a deducir lo que tiempo después ocurriría, la BMV en general comenzó a tener un comportamiento volátil como era de esperarse. 
Teniendo en cuenta que la BMV no se mantuvo ajena a la crisis que se produjo a nivel internacional hemos planteado la siguiente hipótesis nula:

Hipótesis nula: La crisis financiera ocurrida en Estados Unidos, mejor conocida como la Gran Recesión de 2008, habrá provocado un impacto en los precios de las cotizaciones de las empresas de la BMV, puesto que en los mercados financieros internacionales comenzaban turbulencias.

Puesto que la aplicación de la metodología de estudio de eventos requiere la especificación exacta de fechas en las que se producen los acontecimientos que se pretenden analizar, para esta investigación se ha considerado que la Gran Recesión comienza a expandirse cuando se anuncia públicamente la quiebra de Lehman Brothers es decir, el 15 de septiembre de 2008. De hecho Rojas (2009) ha demostrado que a partir de esta fecha se produjeron caídas significativas en los mercados de valores del mundo, al igual que en la BMV.

Derivado de lo anterior y para cumplir con el objetivo planteado se presenta la siguiente hipótesis alternativa.

Hipótesis alternativa: Los precios de los títulos de las empresas que cotizan en la BMV tuvieron un efecto negativo a raíz de la noticia de quiebra del gran banco de inversión Lehman Brothers.

Debido al impacto que tuvo la crisis de Estados Unidos, especialmente con países que tienen relación comercial directa con este, y debido a que México es uno de ellos, tal y como se afirmó en el cuadro 1, entonces los que tardaron más tiempo en resentir la crisis fueron aquellas economías que no se relacionan directamente con la de Estados Unidos, y de igual manera los mercados.

Además se pretende ver si algún sector en particular (energía, materiales, industria, servicios, salud, servicios financieros, o varios) tuvo alguna reacción por la noticia de quiebra del banco Lehman Brothers. De este modo la hipótesis nula a contrastar es la siguiente:

Hipótesis nula: No existe asociación alguna entre los cambios de precios de los títulos de las empresas del sectori que cotizan en la BMV debido a la noticia de quiebra de Lehman Brothers.

\section{Test utilizado para analizar las rentabilidades anormales}

Para contrastar las hipótesis analizadas se llevó a cabo el estudio de las rentabilidades anormales de todas y cada una de las empresas que cotizaron en la BMV para ese periodo. En este análisis se utilizó el test paramétrico estandarizado, donde se trata de analizar la significatividad estadística de los retornos anormales de las empresas cotizadas durante las ventanas evento.

A través del test estadístico estandarizado se pretende analizar la significatividad estadística de los retornos anormales medios y estandarizados (RAM y RAME) sobre los días de la ventana del evento, por cada una de las empresas. Este test permitirá conocer si los RAM y RAME son significativamente diferentes de cero durante toda la ventana del evento. Para cada título $i$ el retorno anormal se estandariza dividiéndolo por su desviación típica estimada, así los retornos anormales estandarizados se calcularon como sigue:

$$
R A E_{i t}=R A_{i t} / S_{i t}
$$


Donde:

$R A E_{i t}=$ retorno anormal estandarizado del título $i$ en el momento $t$

$R A_{i t}=$ retorno anormal del título $i$ en el momento $t$

$S_{i t}=$ desviación típica estimada

Para calcular la desviación típica estimada:

$$
S_{i t}=\left[\alpha_{i}^{2}\left\{1+\frac{1}{T}+\frac{\left(R_{m t}-R_{m}\right) 2}{\sum_{k=t}^{r}\left(R_{m t}-R_{m}\right) 2}\right\}\right] 0.5
$$

Donde:

$S_{i t}=$ desviación típica estimada

$R_{m k}=$ rentabilidad del mercado en los días $k$ del periodo

$R_{m t}=$ rentabilidad del mercado en los días $t$ del evento

$\alpha_{i}^{2}=$ varianza residual del modelo del mercado

La media de los retornos anormales estandarizados por cada día del evento $t$, es el cociente entre la sumatoria de los residuos anormales estandarizados de cada título en el día del evento. Por lo tanto, el test estadístico estandarizado se obtiene:

$$
W_{t}=\overline{R A E_{t} \sqrt{N}_{t}}
$$

Donde:

$R A E_{t}=$ retorno anormal estandarizado

$\sqrt{N}_{t}$ número de días del periodo de estimación

La significatividad estadística de los retornos anormales medios acumulados durante $m$ días desde $t$ hasta $t+m$ se estima utilizando el test estadístico:

$$
W_{t, t+m}=\sum_{r=t}^{N t} W t / \sqrt{m}
$$

Asumiendo que los retornos anormales son independientes a través del tiempo $W_{(t, t+m)}$ también se distribuye como una normal unitaria. Por lo tanto, ambos estadísticos permiten contrastar si los retornos estandarizados medios para el día $t$ y los retornos anormales estandarizados medios acumulados, de todas las empresas son iguales a cero o no.

\section{Análisis empírico aplicado a las empresas que cotizan en la BMV}

La muestra inicial está formada por todas las empresas cotizadas en el mercado continuo mexicano, mercado que opera por concesión de la Secretaría de Hacienda y Crédito Público, y que se rige por la Ley del Mercado de Valores. Sus accionistas son exclusivamente las casas de bolsa autorizadas, las cuales poseen una acción cada una. Las operaciones y negociaciones de valores del mercado de capitales se realizan en la plataforma tecnológica desarrollada y administrada por la Bolsa Mexicana de Valores. El sistema totalmente descentralizado y automatizado, permite negociar valores en tiempo real, a través de cientos de terminales de computadoras interconectadas por una red, ubicadas en las casas de bolsa y controladas por la estación de Control Operativo de la BMV. 
El análisis se llevó a cabo con 87 empresas de las 143 inscritas, de las cuales se obtuvo información de las cotizaciones diarias durante un período comprendido de enero de 2008 a febrero de 2009. Por lo tanto, se obtuvieron cotizaciones de 302 días para cada una de las empresas, analizando así 26,274 cotizaciones en total para cumplir el objetivo del presente trabajo.

Para que los resultados no fueran sesgados se cuidó que todas cumplieran con los siguientes requisitos:

1) Los ejercicios económicos de las empresas tenían que terminar el 31 de diciembre.

2) Durante los días de la ventana del evento las empresas tenían que haber cotizado en más del $80 \%$ de las sesiones. Con ello se pretende garantizar un número mínimo de observaciones para cada empresa.

Además se realizo la clasificación de las empresas por sector como se muestra en el cuadro 2.

Cuadro 2. Muestra BMV por sector de actividad.

\begin{tabular}{|l|c|}
\hline $\begin{array}{l}\text { Muestra por } \\
\text { sector BMV }\end{array}$ & $\begin{array}{c}\text { Muestra } \\
\text { relativa al } \\
01 / 12 / 2013\end{array}$ \\
\hline Materiales & 23 \\
\hline Industria & 31 \\
\hline Servicios & 27 \\
\hline Salud & 6 \\
\hline Muestra final & $\mathbf{8 7}$ \\
\hline
\end{tabular}

Fuente: Elaboración propia.

Una vez expuesto lo anterior y conocido el procedimiento para la obtención de la muestra, se procede al análisis de los resultados obtenidos.

Ya que han sido calculados los parámetros alfa y beta con el modelo de mercado, para cada una de las empresas de la muestra durante el período de estimación y de acuerdo a los valores de los betas, se presentan los resultados obtenidos con el estadístico estandarizado $W_{t, t+k}$, con el propósito de determinar la significatividad estadística de los retornos anormales medios acumulados durante las distintas ventanas evento para las empresas mencionadas. Véase cuadro 3. 
Cuadro 3. Valores del estadístico estandarizado para la fecha de evento

\begin{tabular}{|c|c|c|c|c|}
\hline Días & RAETM & Wt & $W_{t, t+1}$ & Días evento \\
\hline-10 & -0.13889942 & -1.29556757 & $4.48483548^{\star \star \star}$ & 21 días $-10,+10$ \\
\hline-9 & 0.07763403 & 0.72412202 & $4.46576114^{\star \star \star}$ & 19 días $-9,+9$ \\
\hline-8 & 0.04779714 & 0.44582206 & $4.01023420^{\star \star \star}$ & 17 días $-7,+7$ \\
\hline-7 & -6.89713005 & -0.00064332 & $3.60972292^{\star \star \star \pi}$ & 15 días $-6,+6$ \\
\hline-6 & 0.04635648 & 0.43238443 & $3.44103798^{\star * *}$ & 13 días $-5,+5$ \\
\hline-5 & 0.08813623 & 0.82208006 & $3.05505708^{* * *}$ & 11 días $-5,+5$ \\
\hline-4 & 0.07058037 & 0.6583299 & $2.56084419^{\star * *}$ & 9 días $-4,+4$ \\
\hline-3 & -0.06031292 & -0.56256144 & $2.04058017^{\text {*** }}$ & 7 días $-3,+3$ \\
\hline-2 & -0.11481009 & -1.07087721 & $1.84017086^{*}$ & 5 días $-2,+2$ \\
\hline-1 & 0.09386416 & 0.87550664 & $2.20286793^{* *}$ & 3 días $-1,+1$ \\
\hline 0 & 0.15949454 & 1.48766599 & 1.48766599 & día 0 \\
\hline 1 & 0.15570361 & 1.45230655 & & \\
\hline 2 & 0.14689498 & 1.37014515 & & \\
\hline 3 & 0.19798509 & 1.84668197 & & \\
\hline 4 & 0.1742542 & 1.62533501 & & \\
\hline 5 & 0.17452549 & 1.62786541 & & \\
\hline 6 & 0.1974806 & 1.84197641 & & \\
\hline 7 & 0.16877208 & 1.5742012 & & \\
\hline 8 & 0.22604425 & 2.10840037 & & \\
\hline 9 & 0.2366217 & 2.20706033 & & \\
\hline 10 & 0.25536264 & 2.38186411 & & \\
\hline
\end{tabular}

La significatividad del estadístico al $1 \%$ se da a partir de 2.56

La significatividad del estadístico al $5 \%$ se da a partir de 1.96

La significatividad del estadístico al $10 \%$ se da a partir de 1.645

$* * *$ Significatividad al $1 \%$

**Significatividad al $5 \%$

*Significatividad al 10\%

Fuente: Elaboración propia.

Como se puede observar en el cuadro anterior, el test estandarizado se aplicó a los retornos anormales de las empresas inscritas en la BMV. El test se utilizó para diferentes ventanas de evento, es decir, para 21 días $(-10,+10)$, para 19 días $(-9,+9)$, para 17 días $(-7,+7)$, y así sucesivamente incluido el día del evento (día cero).

Si las empresas inscritas en la BMV hubieran sufrido una disminución de los precios tras conocerse la noticia de la quiebra de Lehman Brothers, el análisis de los RAM tendría que haber arrojado resultados significativamente diferentes de cero para las empresas. Los resultados nos ofrecieron valores estadísticamente significativos al $1 \%$ para las ventanas que van desde $(-4,+4)$ hasta $(-10,+10)$. Sin embargo, las rentabilidades anormales nos mostraron resultados negativos, lo cual significa que efectivamente el mercado no penalizó a las empresas cuando el evento fue conocido, el día cero muestra un valor no significativo. Habría que resaltar que el valor de las acciones disminuyó conforme se acerca el día del evento, sin embargo los resultados no muestran resultados negativos para ninguna de las ventanas. Para sustentar la disminución en el precio de las acciones se puede observar la gráfica 1 en la que se visualiza la disminución en las rentabilidades anormales en los días previos y en la fecha del evento. 
Gráfico 1. Comportamiento de los rendimientos de las empresas duante las ventanas del evento.

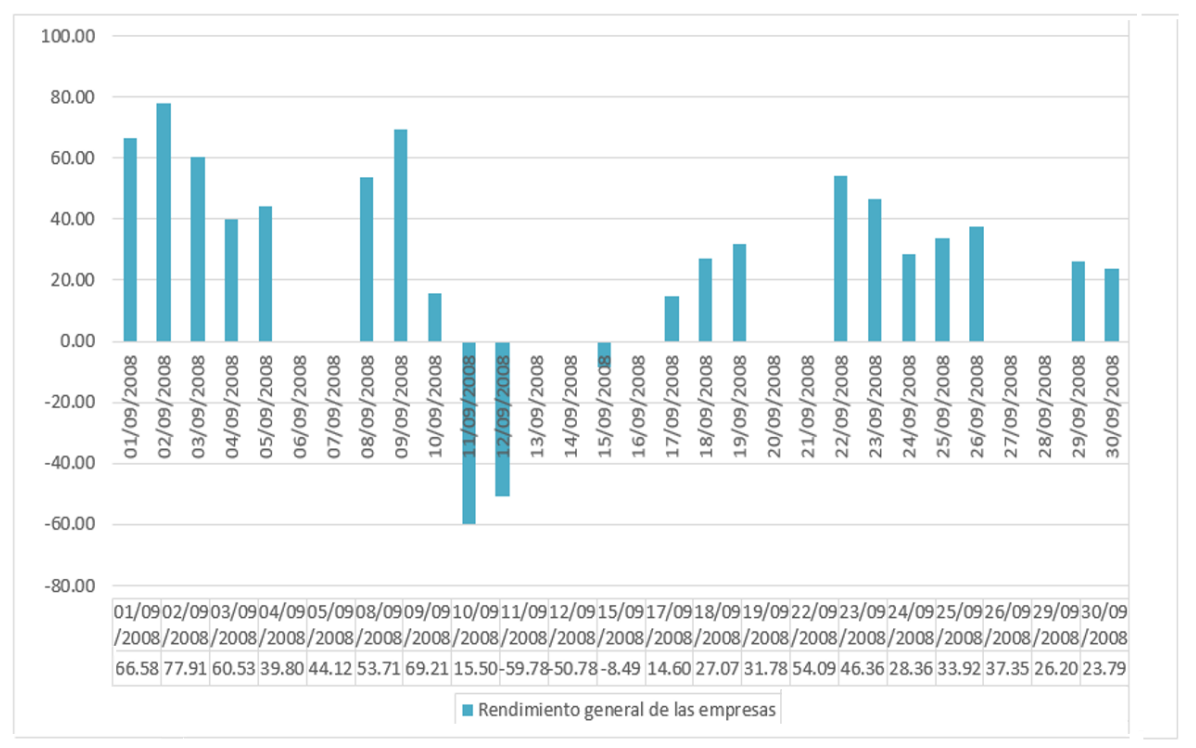

Fuente: Elaboración propia.

Como se puede observar claramente en los rendimientos de las empresas, es tres y cuatro días respectivamente antes a la fecha del evento cuando éstos comienzan a verse negativos, lo que claramente significaría que una vez que se anuncia la quiebra de Lehman Brothers el mercado comienza a reaccionar de manera negativa, pero esto comienza desde el 10 de septiembre cuando existe un rendimiento menor al que se ha presentado días anteriores (9 y 8) recuperándose casi una semana después, pero con rendimientos claramente bajos. Es hasta el 22 de septiembre cuando se comienza a recuperar, pero aún con algunas turbulencias para repuntar. Ahora bien, hay que analizar la reacción de los sectores de actividad de la BMV, para determinar el efecto sobre cada uno de estos.

\section{Resultados sobre las empresas que cotizan en la BMV clasificadas por sector de actividad}

Con la finalidad de dar mayor solidez a la investigación se ha desarrollado un análisis por sector de actividad, con el cual se pretende descubrir si algún sector en particular se vio afectado por la noticia de quiebra de Lehman Brothers. Así, se ha utilizado para el estadístico $W_{t, t+m}$ las mismas ventanas del análisis anterior. Los resultados presentados pretenden demostrar si hay o no asociación entre los cambios de los precios de los títulos de algún sector específico y el evento analizado. En el siguiente cuadro se presentan los resultados obtenidos en el estadístico para el sector industrial y el sector materiales en la fecha en la que Lehman Brothers se declara en quiebra. 
78 Nueva Época REMEF (The Mexican Journal of Economics and Finance)

Cuadro 4. Valores de las pruebas paramétricas y no paramétricas para la fecha de evento 15 de septiembre de 2008 en los sectores industrial y materiales.

\begin{tabular}{|c|c|c|c|c|c|c|c|c|c|}
\hline "Días & Sector & RAETM & Wt & $W_{t, t+1}$ & Sector & RAETM & Wt & $W_{t, t+1}$ & Dias evento \\
\hline-10 & \multirow{21}{*}{ 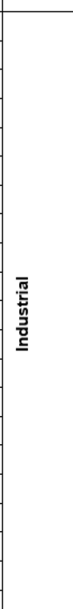 } & -0.38795544 & -2.16004447 & $-2.5704855^{\star \star \star}$ & \multirow{21}{*}{ 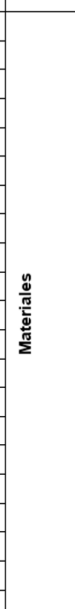 } & -0.1315182 & -0.68338863 & $6.21004697^{\star \star \star}$ & 21 dias $-10,+10$ \\
\hline-9 & & -0.05628019 & -0.52494669 & $-2.13714472^{\star \star}$ & & 0.32810014 & 1.70485835 & $6.30606315^{\star \star \star \star}$ & 19 días $-9+9$ \\
\hline-8 & & -0.07351025 & -0.68565792 & $-2.04648268^{* \pi}$ & & 0.25669937 & 1.33384907 & $5.95475098^{\star \star \star *}$ & 17 días $-7,+7$ \\
\hline-7 & & -0.10036436 & -0.93613646 & $-1.90453543^{\star \star}$ & & 0.19825097 & 1.03014228 & $5.65075326^{* \star *}$ & 15 dias $-6,+6$ \\
\hline-6 & & -0.06738903 & -0.62856306 & $-1.70024557^{\star}$ & & 0.29223043 & 1.51847387 & $5.51638472^{\star \star \star}$ & 13 días $-5,+5$ \\
\hline-5 & & -0.05614686 & -0.52370308 & -1.5519233 & & 0.36599459 & 1.90176369 & $5.02666599^{* \star \star}$ & 11 dias $-5,+5$ \\
\hline-4 & & -0.05317626 & -0.49599514 & -1.39124142 & & 0.29997047 & 1.55869227 & $4.15627133^{\star \star \star}$ & 9 días $-4,+4$ \\
\hline-3 & & -0.10072601 & -0.93950971 & -1.30456051 & & 0.0994707 & 0.5168649 & $3.44470145^{\star \star \star}$ & 7 dias $-3,+3$ \\
\hline-2 & & -0.11291307 & -1.05318303 & -0.96909662 & & -0.04776602 & -0.24819951 & $2.71405783^{\star \star \star \star}$ & 5 días $-2,+2$ \\
\hline-1 & & -0.02977199 & -0.27769459 & -0.43841837 & & 0.27075267 & 1.40687212 & $2.63411535^{\text {kᄎ }}$ & 3 dias $-1,+1$ \\
\hline 0 & & -0.01971299 & -0.1838705 & -0.1838705 & & 0.32231471 & 1.67479638 & $1.67479638^{\star}$ & dia 0 \\
\hline 1 & & -0.03192728 & -0.2977978 & & & 0.28497107 & 1.48075312 & & \\
\hline 2 & & -0.03799781 & -0.35442001 & & & 0.3376721 & 1.75459569 & & \\
\hline 3 & & -0.03699507 & -0.34506703 & & & 0.4865409 & 2.52814068 & & \\
\hline 4 & & -0.02424973 & -0.22618644 & & & 0.34569778 & 1.79629836 & & \\
\hline 5 & & -0.04821504 & -0.44971997 & & & 0.44282522 & 2.30098733 & & \\
\hline 6 & & -0.03801842 & -0.35461224 & & & 0.32708222 & 1.69956907 & & \\
\hline 7 & & -0.03321136 & -0.30977494 & & & 0.185815 & 0.96552304 & & \\
\hline 8 & & -0.04030847 & -0.37597235 & & & 0.25652537 & 1.33294492 & & \\
\hline 9 & & -0.03782273 & -0.35278692 & & & 0.23682261 & 1.23056637 & & \\
\hline 10 & & -0.032571 & -0.30380205 & & & 0.31829455 & 1.65390698 & & \\
\hline
\end{tabular}

La significatividad del estadístico al $1 \%$ se da a partir de 2.56

La significatividad del estadístico al $5 \%$ se da a partir de 1.96

La significatividad del estadístico al $10 \%$ se da a partir de 1.645

$* * *$ Significatividad al $1 \%$

**Significatividad al $5 \%$

*Significatividad al $10 \%$

Fuente: Elaboración propia.

Para observar el efecto en el sector industrial por la Gran Recesión, el estadístico en la ventana $(-10,+10)$ nos muestra que los RAM son estadísticamente significativos al $1 \%$ y los valores son negativos, sin embargo en los valores para las ventanas $(-1,+1),(-2,+2),(-3,+3),(-4,+4)$ no se muestran diferencias estadísticamente significativas, lo que claramente nos lleva a aceptar que las empresas de este sector se vieron penalizadas dada la noticia de quiebra de Lehman Brothers, como se observa. Tanto el estadístico, como los RAM para este sector nos muestran resultados negativos lo que llevaría a suponer claramente que por consecuencia las empresas de este sector que cotizan en la BMV generaron una desconfianza para los inversionistas.

Por otro lado, el cuadro nos permite observar que para el sector materiales se muestran resultados estadísticamente significativos, al $1 \%$ para todas las ventanas establecidas, y al 10\% para el día cero, es decir el día del evento, lo que nos deja concluir que efectivamente hubo efectos este día, sin embargo dichos resultados son positivos, situación contraria a la esperada cuando la crisis ya está presente. Asimismo, y prosiguiendo con el rumbo de la investigación se presentan en el siguiente cuadro los resultados para los sectores servicios y salud, respectivamente. 


\section{Cuadro 5. Valores de las pruebas paramétricas y no paramétricas para la} fecha de evento 15 de septiembre de 2008 en los sectores servicios y salud.

\begin{tabular}{|c|c|c|c|c|c|c|c|c|c|}
\hline Días & Sector & RAETM & Wt & $W_{t, t+1}$ & Sector & RAETM & Wt & $W_{t, t+1}$ & Dias evento \\
\hline-10 & \multirow{21}{*}{ 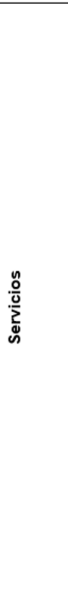 } & -0.07843593 & -0.73160167 & $2.82223323^{* \star *}$ & \multirow{21}{*}{ 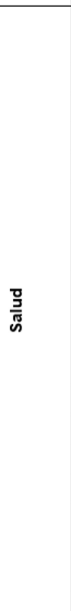 } & 4.16848259 & 10.2106554 & 48.2289201 & 21 días $-10++10$ \\
\hline-9 & & 0.04919474 & 0.45885797 & $2.75388449^{\star \star \star}$ & & 4.2049971 & 10.3000973 & 45.9270525 & 19 días $-9,+9$ \\
\hline-8 & & 0.02723196 & 0.25400281 & $2.36967877^{x \star}$ & & 4.22555951 & 10.3504647 & 43.4803718 & 17 días $-7,+7$ \\
\hline-7 & & -0.00075729 & -0.00706358 & $2.02928444^{* \star}$ & & 4.24103121 & 10.3883624 & 40.865144 & 15 dias $-6,+6$ \\
\hline-6 & & 0.00689095 & 0.06427453 & $1.80702187^{\star}$ & & 4.25374656 & 10.4195086 & 38.0552694 & 13 días $-5,+5$ \\
\hline-5 & & 0.03036787 & 0.28325264 & 1.51879613 & & 4.26204376 & 10.4398325 & 35.0124682 & 11 días $-5,+5$ \\
\hline-4 & & 0.0191556 & 0.17867157 & 1.27881671 & & 4.26478916 & 10.4465573 & 31.6686589 & 9 dias $-4,+4$ \\
\hline-3 & & -0.08329459 & -0.77692022 & 0.9021365 & & 4.24393296 & 10.3954703 & 27.92232 & 7 días $-3,+3$ \\
\hline-2 & & -0.06992136 & -0.65218302 & 0.93731656 & & 4.24794235 & 10.4052912 & 23.6248624 & 5 dias $-2,+2$ \\
\hline-1 & & 0.03414747 & 0.31850636 & 1.09182101 & & 4.31576475 & 10.5714215 & 18.3606297 & 3 dias $-1,+1$ \\
\hline 0 & & 0.0814675 & 0.7598783 & 0.7598783 & & 4.33840214 & 10.6268716 & 10.6268716 & día 0 \\
\hline 1 & & 0.0871311 & 0.81270481 & & & 4.32875887 & 10.6032505 & & \\
\hline 2 & & 0.09187973 & 0.85699709 & & & 4.33558195 & 10.6199635 & & \\
\hline 3 & & 0.11448505 & 1.0678455 & & & 4.34916962 & 10.6532464 & & \\
\hline 4 & & 0.13626011 & 1.27094973 & & & 4.36168577 & 10.6839045 & & \\
\hline 5 & & 0.09837427 & 0.91757412 & & & 4.3590347 & 10.6774108 & & \\
\hline 6 & & 0.15157083 & 1.41375859 & & & 4.3549872 & 10.6674965 & & \\
\hline 7 & & 0.14485724 & 1.35113841 & & & 4.35659496 & 10.6714347 & & \\
\hline 8 & & 0.17765422 & 1.65704824 & & & 4.34934612 & 10.6536787 & & \\
\hline 9 & & 0.1902582 & 1.77461035 & & & 4.33442031 & 10.6171181 & & \\
\hline 10 & & 0.1780559 & 1.66079489 & & & 4.33177599 & 10.6106409 & & \\
\hline
\end{tabular}

La significatividad del estadístico al $1 \%$ se da a partir de 2.56

La significatividad del estadístico al $5 \%$ se da a partir de 1.96

La significatividad del estadístico al $10 \%$ se da a partir de 1.645

$* * *$ Significatividad al $1 \%$

**Significatividad al $5 \%$

*Significatividad al $10 \%$

Fuente: Elaboración propia.

En el cuadro anterior, para el sector servicios se observa que los resultados muestran significatividad al $1 \%$ para las ventanas $(-10,+10)$ y $(-9,+9)$ respectivamente, sin embargo dichos resultados son positivos lo que nos lleva a rechazar que estas empresas hayan tenido contracciones negativas para la fecha del evento analizado, prosiguiendo, en el mismo cuadro presentamos los resultados para el sector salud, aunque fácilmente se puede afirmar que este sector por ser el que posee menor número de emisoras es el que menos efectos tuvo.

Ahora bien, como consideraciones finales el objetivo de este trabajo ha sido ofrecer evidencia empírica sobre los efectos económicos de la quiebra del gran banco de inversión. En concreto, se ha estudiado si la denominada Gran Recesión ha afectado los precios en los títulos de las empresas que cotizan en la BMV.

Es por lo anterior que la denominada "Gran Recesión", constituye una oportunidad única para tratar de abordar este tema, dadas las enormes consecuencias que ha tenido no sólo en Estados Unidos, sino en el resto de los países, en México se dejaron sentir las consecuencias de este fenómeno.

En el análisis de las cotizaciones del mercado continuo mexicano, los resultados obtenidos a través de la utilización de la metodología de eventos, no 
muestran una reacción negativa significativa desde el punto de vista estadístico, excepto para el sector industrial, donde los resultados fueron negativos y significativos por lo tanto los resultados de nuestro trabajo no pueden confirman la teoría de que el mercado mexicano reaccionó de manera totalmente negativa, tal y como lo mencionan varios autores (véase entre otros Rojas, 2009 y Parodi, 2013)

Los datos estadísticos obtenidos en el trabajo no nos permiten aceptar en su mayoría la hipótesis alternativa planteada, es decir la reacción no se traduce en penalización a las empresas a causa de la Gran Recesión.

Sin embargo, sí hubo reacción negativa en el sector industrial, lo cual nos llevaría a pensar que este sector fue el de mayor peso en los movimientos generales del mercado mexicano como se visualizó en la gráfica anterior, asimismo se puede deducir que fue este el sector más afectado debido a que todo el desastre se dio con las hipotecas subprime, y dado que es este sector el más relacionado a la construcción, y las empresas cementeras son las que aquí se encuentran inscritas.

Finalmente, se ha de mencionar que los resultados deben ser interpretados con prudencia. La primera limitación de este estudio radica en el número de empresas de las cuales se pudo obtener información de cotización diaria, ya que no fueron todas las que se encuentran inscritas como se tenía pensado, algunas no cotizaron para esas fechas, y otras se inscribieron después de la fecha del evento analizado. En segundo lugar, cabe mencionar como posible limitación la elección de los períodos de estimación y ventanas evento que pueden variar de acuerdo a las consideraciones de cada investigador.

\section{Conclusiones}

Las crisis en general han conducido a la población total a vivir con miedo y resaca económica, es decir ha de haber llegado el momento en que las personas ya "se acostumbraron" a vivir estos fenómenos, de tal manera que, especialmente las personas de la clase social media baja y baja resienten de manera directa y concreta, sin embargo, esto no quiere decir que fenómenos como el analizado en este trabajo no se puedan evitar, más bien se deben tomar investigaciones como esta para defenderse frente a sucesos posteriores, es decir, se busca que esta sea una herramienta que sirva de apoyo para gobierno y empresas, y en lugar de quedarse en la "comodidad", utilizar la situación de crisis para sacar provechos y obtener progresos tal y como puntualizó Einstein.

Ahora bien, el objetivo de este trabajo fue ofrecer evidencia sobre los posibles efectos económicos de la crisis ocurrida en 2008, en concreto, se ha estudiado y obtenido evidencia para determinar si la denominada "Gran Recesió" ha afectado las cotizaciones bursátiles de las empresas que cotizan en la BMV.

Dicha evidencia se obtuvo mediante la elaboración de un estudio a través del cual se ha podido conocer la reacción del mercado de las empresas mexicanas que cotizan en la BMV, asimismo se puede afirmar que este trabajo además de ofrecer las evidencias mencionadas, expone fundamentos teóricos, pues los análisis y estudios realizados están sustentados en una revisión de artículos y autores especializados en la materia.

Tal y como se menciona en el desarrollo de la investigación, las crisis 
financieras no sólo afectan al país epicentro, si no que se trata de una epidemia que como su nombre lo dice, comienza a propagarse de manera rápida a los alrededores, afectando así, en primera instancia a los países con relación comercial directa, y estos a su vez con aquellos más cercanos, para comprender mejor un fenómeno de esta índole hay que recalcar que una crisis financiera puede provocar lo que se llama "efecto domin" dejando huella alrededor de todo el mundo.

Para mitigar estos efectos, particularmente en México lo que varios autores recomiendan, y de manera personal se apoya, es que se busquen otras fronteras para tener relaciones comerciales, si esto se hace una crisis financiera ocurrida en el país vecino ya no afectaría tan directamente a México, cabe mencionar que las crisis y escándalos más grandes del mundo han tenido lugar en Estados Unidos. Para determinar el grado de efecto del fenómeno analizado en el mercado mexicano, es necesario establecer qué otras cosas o acontecimientos provocan los cambios en las cotizaciones, entre las cuales se encontraron: los tipos de interés, los beneficios de la empresa, la estabilidad económica y monetaria, entre otras, siendo esta última la que más altamente afecta al mercado mexicano, encontrándose dentro de este punto fenómenos como crisis. Entonces fue la crisis de 2008, junto con las variantes mencionadas las que sucumbieron el pánico en la economía mundial y en las bolsas más importantes del mundo, cabe recalcar que no se deben dejar de lado el grado de impacto de las otras variables mencionadas.

Los resultados del estudio empírico demuestran que la noticia de quiebra del banco de inversión estadounidense Lehman Brothers tuvo consecuencias sobre la rentabilidad de los títulos de algunas de las empresas que cotizan en la Bolsa Mexicana de Valores. En este mercado se negocian acciones representativas de las empresas inscritas dentro de la BMV, mismas que juegan un papel importante dentro del sector productivo de la economía, pero que sin embargo, en varias de las ocasiones el desempeño de éstas no influye en el comportamiento del precio de las acciones, debiéndose básicamente a otro tipo de eventos, tal y como ha venido sucediendo desde el inicio de la crisis financiera. Así el rendimiento experimentado por la BMV desde el día 1 de agosto de 2007 al 16 de julio de 2009 ha sido del (-16.06\%), pérdida importante, pero menor si tomamos en consideración que al 27 de octubre de 2008, día en el que el IPC llegó a su nivel mínimo el rendimiento era de (-44.98\%) (Rojas, 2012).

Así, observamos que mientras el IPC experimenta una contracción de $22.56 \%$ desde el 1 de agosto de 2007, 28 acciones experimentaron rendimientos positivos en el mismo periodo, dentro de las cuales destacan de las demás ELEKTRA, AUTLAN Y MINSA, acciones que han experimentado rendimientos superiores al $100 \%$, aunque las dos primeras en un porcentaje mucho mayor que la tercera, de igual manera se puede decir que no existe un patrón que defina el sector específico que ha presentado resultados positivos, ya que estas empresas son de giro distinto.

En lo que respecta a las acciones que al igual que el IPC, han experimentado un rendimiento negativo durante este periodo de crisis, destaca el hecho de que haya sido el sector industrial el que se vio afectado de manera importante, asimismo dentro de estas acciones se encuentran las relacionadas a la construcción de vivienda como es el caso de HOGAR quien experimentó una 
contracción de $82.97 \%$, SARE (80.05\%), ARA (65.35\%), GEO (54.68\%), URBI $(52.63 \%)$ o bien las de cemento como CEMEX (66.51\%) y GCC $(55.81 \%)$.

Aunque la acción que experimentó la mayor pérdida desde el inicio de la crisis pertenece a una emisora del sector servicios, (Citigroup) la cual tuvo una contracción de $91.83 \%$. Destacan dentro de este comportamiento negativo, acciones como AXTEL quien experimentó pérdidas en su precio de $90.99 \%$, o el de la acción GISSA, con una contracción de $86.24 \%$.

De todo lo anterior fácilmente se puede observar que el sector afectado en mayor grado fue el sector construcción, esto debido a que el sector inmobiliario fue el detonante de la crisis financiera ocurrida en el 2008, entonces fue esta la razón por la cual empresas dedicadas a la construcción son las que se vieron influenciadas en primera instancia.

Finalmente y para cerrar la investigación cabe hacer énfasis en que son este tipo de investigaciones las que deben ser analizadas por quienes tienen el poder de evitar un fenómeno como el ocurrido, así pues también cabe señalar que los inversores no deben dejarse vencer por el pánico de una caída de precios, ya que casi todas las veces este pánico es el que realmente hace que haya un cambio drástico.

También y como recomendación personal al público lector de la investigación es pertinente mencionar que en situaciones como lo sucedido en 2008, lo más conveniente para todos es actuar con cautela, y no dejarse llevar por el pánico que sucumbe en esos momentos, ya que si esto se hace de manera eficiente claramente se obtendrán beneficios, asimismo y para puntualizar lo que Einstein mencionó "la crisis es la mejor bendición que puede sucederles a las personas y paises, porque la crisis trae progreso".

\section{Bibliografía}

Aguilar, R. (2008). La crisis y la economía real. Periódico el Financiero, (4 de noviembre, 2008). pp. 26.

Arce, R., Mahía, R., Pérez, J., and Vícens, J. (1988). Informe sobre los efectos de la crisis asiática en las economías occidentales.

Abreu. (2011). Crisis y paquete de rescate financiero de EE.UU., Coyuntura política nacional e internacional, Economía, mayo 2011, disponible en https://coyunturapoliticamx.word _press.com/2011/05/10/crisis-y-paquete-de-rescate-financiero-de-ee-uu/.

Banamex. (2008). Examen de la situación económica. Estudios económicos y sociales, pp. 281-332.

BBC. (2011). La crisis financiera de 2008 en estados unidos habría podido evitarse, BBC Mundo.com, 27 de enero de 2011, disponible en http://www.bbc.com/mundo/noticias /2011/01/110127_economia_eeuu_crisis_evitable_jrg.shtml?print=1, consultado el 11 de mayo de 2015.

BBC. (2008). Cronología de una crisis, BBC Mundo.com, 15 de septiembre de 2008, disponible en http://news.bbc.co.uk/hi/spanish/specials/2008/crisisfinanciera/newsid $-7315000 / 7315229$. stm.

Castellanos,R. (2004). La crisis económica global en los marcos de la globalización neoliberal. Revista Santiago, 105 (1), pp. 114-126.

Córdoba, M. (2010). ¿Bonanza o burbuja en américa latina y el caribe? Qué se aprendió de la crisis financiera global? Nuevos desafíos para el desarrollo de américa latina y el caribe. XXIII seminario regional de política fiscal, CEPAL. Santiago, Chile.

Discovery Channel. 7C Producciones. Periodista: El Club Bildelberg. Producción y Edición, Marco Antonio Chile, (2012). 
Dodd, R. (2007). Los tentáculos de la crisis hipotecaria en finanzas y desarrollo, Fondo Monetario Internacional, pp. 15-19.

FMI. (2015). Perspectivas de la economía mundial. Octubre, 2015, pp.154-182

García, M. (2008). Efecto de la crisis financiera en los mercados bursátiles. Foro de iniciativas y negocios. Club financiero Vigo 14a ed. pp. 1-4.

García, R. (2008). Las cuatro crisis concurrentes. Periódico el Financiero, (06 de octubre), pp. 18.

Girón, A. (2002). Crisis financiera, textos breves de economía de miguel ángel. Porrúa librero-editor y el Instituto de Investigaciones Económicas, UNAM, México.

Gitman, L. y Mc Daniel, C. (1995). El mundo de los negocios. Ed. Harla, México. pp 51-55.

Gómez, J. y Pérez, F. (2004). Stock Market Cycles and Stock Market Development in Spain. Spanish Economic Review, 6, pp. 127-151.

Hernández, F. (2009). La crisis financiera de 2008: ¿De dónde viene? Revista de Historia Internacional, 9(36). Centro de Investigación y Docencia Económicas. pp. 73-86.

Jaramillo, M. y García, M. A. (2010). Implicaciones de los escándalos financieros: evidencia para méxico. Santander, España.

Jaramillo, M. y García, M. A. (2012). Reacción del mercado de valores mexicano ante los escándalos financieros: Evidencia empírica. Revista Mexicana de Economía y Finanzas Nueva época, 7(2), pp. 129-153.

Melo, L. y Rincón, H. (2012). Choques externos y precios de los activos en latinoamérica antes y después de la quiebra de lehman brothers. Borradores de economía. Bogotá, Colombia.

Mackinlay, R. (1997). Event Studies in Economics and Finance. Journal of Economic Literature, 35 (1), pp. 13-39.

OCDE. (2015). Economic Policy Reforms: Going for Growth

Ruíz, R. y De La Torre, A. (2007). Determinación y análisis de la tendencia y el ciclo en las series bursátiles. Análisis Financiero, 1004(2) pp. 14-23.

Ramírez, R. (2012). México en la crisis global y en su propia crisis: qué hacer? Ensayo para la Fundación Friedrich Ebert.

Samuelson, P. y Nordhaus, W. (2002). Economía. Ed. Mc Graw Hill. 17 edición, pp. 455-465.

Sandoval, A. (2008). La bolsa mexicana de valores, cerca del fin del ciclo virtuoso. Periódico El Financiero, sección análisis económico, enero 7 de 2008, pp. 3.

Shiller, R. (2005). Irrational Exuberance. Second Edition, Princeton University Press, Princeton.

SER. (2010). La crisis financiera en EE.UU. y sus implicaciones para méxico. Informe de la Secretaría de Relaciones Exteriores.

Tomé, J.P. (2013). La crisis económica mundial y la acumulación de capital, las finanzas y la distribución del ingreso. Debates en la economía marxista. Universidad Pontificia Comillas. Madrid, España.

Vito, T. (2009). the economic Role of the State before and after the Current Crisis. Available in: http://www.iipf.net/speeches/Tanzi_2009.pdf 